\title{
Une naissance monstrueuse au Moyen Age: le «frère de Salerne»
}

Mireille Ausécache

\section{Summary}

Gilles de Corbeil (XIIth century) reports the strange medical case of the "Salernitan brother", a monster born together with a normal child. This disorder is said to be typical of Salernitan women. The phenomenon which is still commented on by physicians in the following centuries is rather difficult to explain for it seems to depend on both scholarly medicine and popular beliefs.

Keywords: Salernitan medicine; monstrous birth; Gilles de Corbeil; medieval gynaecology

\section{Résumé}

Gilles de Corbeil (XII s.) rapporte l'étrange cas du «frère de Salerne», monstre accompagnant parfois la naissance d'un enfant normal. Cette maladie est présentée comme spécifique aux femmes de la région de Salerne. Le phénomène encore commenté par les médecins aux siècles suivants est difficile à interpréter tant il semble se situer à la frontière entre médecine savante et croyances populaires.

Mireille Ausécache, 6, route de Verdon, F-02330 Pargny-la-Dhuys (mireilleausecache@ wanadoo.fr). 


\section{Dans son long traité versifié sur les «Vertus des médicaments composés» écrit}

à la fin du XII ${ }^{\mathrm{e}}$ siècle, le médecin français Gilles de Corbeil ${ }^{1}$, commentant les vertus innombrables de la Thériaque ${ }^{2}$, nous livre un bien curieux témoignage (livre IV, vers 666-719):

La thériaque expulse le foetus mort et le frère de Salerne, monstre dégénéré par sa forme, anormal quant à sa conception, semblable au goret nouveau-né ${ }^{3}$ mais plus terrifiant par nature et effrayant par la vue. Chez les femmes samnites ${ }^{4}$, il précède parfois l'accouchement, devançant l'enfant qui était son compagnon dans le ventre maternel. [...] Le père italien éprouve par cela sa paternité et l'avenir de sa lignée; la femme se réjouit de cet événement et supporte d'autant mieux la douleur de l'enfantement qu'elle rachète une faute passée. Elle ose alors couronner sottement de louanges son honneur, elle qui peut si facilement se laisser séduire par un étranger (francus) de passage ou par le marchand ambulant tant apprécié des femmes. La mère périt subitement si le corps nu de la bête difforme touche la terre: le sol de la maison est alors recouvert de tissus et l'animal est tué à coups de bâton. Cette tare de la nature, cette honte pour l'espèce humaine, cette colère tombée du ciel a été transmise à Salerne par les habitants de Bénévent pour faire injustement partager leur déshonneur à la population de cette ville dont la réputation resplendit dans le monde entier grâce à la qualité de sa médecine. L'origine du monstre peut être la corruption du sperme, la consommation d'herbes, l'abondance des menstrues, un dérèglement de la matrice ou l'effet d'une sentence divine pour une faute passée. La médecine peut anéantir le mal et en éloigner la cause grâce aux épices, herbes et aromates; un culte régulier à Saint Mathieu peut apaiser la colère du ciel. ${ }^{5}$

\section{Les différents aspects de ce texte laissent perplexe tant semblent mêlées} des considérations d'ordre différent: aspect médical d'abord d'un phéno-

1 Ce médecin français formé à Salerne, est l'auteur de quatre traités médicaux en vers: $D e$ urinis, De pulsibus, De uirtutibus et laudibus compositorum medicaminum, Viaticus de signis et symptomatibus aegritudinum et d'un poème satirique à l'encontre du clergé, la Hierapigra ad purgandos prelatos. Sa biographie est mal connue mais on pense qu'il vécut entre 1140 et 1220 et qu'il fut l'un des médecins de Philippe Auguste. Cf. Vieillard 1908. Ses traités des urines et du pouls connurent un grand succès et figurent dans de nombreux manuscrits, assortis souvent de commentaires. Ils furent également imprimés très tôt. Ses autres œuvres n'eurent pas la même fortune et il faut attendre le XVIII ${ }^{e}$ siècle pour voir le traité des médicaments imprimé dans un recueil de poésie latine édité par P. Leyser; il fut réédité par L. Choulant en 1826. Le traité des médicaments est à la fois une œuvre médicale et littéraire où sont commentées les vertus de 80 médicaments composés, commentaire agrémenté de nombreuses digressions qui présentent souvent un grand intérêt. Cf. Ausécache 2003. Sur Salerne voir notamment Kristeller 1956, 1986, 1993.

2 Célèbre préparation élaborée à partir du $\mathrm{II}^{\mathrm{e}}$ siècle av. J.-C. d'abord par Nicandre puis Mithridate, elle fut enrichie par les médecins de Trajan puis de Néron jusqu'à contenir 71 substances. Conçue d'abord pour traiter les morsures de bêtes sauvages, elle fut considérée comme une panacée jusqu'au XVIII ${ }^{\mathrm{e}}$ siècle et figura dans les traités d'officine jusqu'au XIX ${ }^{\mathrm{e}}$ siècle, étant alors recommandée pour les affections des voies digestives en raison de ses composants opiacés. Prescrite également pour provoquer les menstrues, elle peut entrer dans la préparation de remèdes abortifs.

3 Les connaissances salernitaines en matière d'anatomie sont largement tributaires de la dissection du porc, ce qui fait de cet animal une référence fréquente. Cf. de Renzi II 1852-1857, 387-401.

4 Ce terme veut sans doute simplement désigner les femmes de la région de Salerne, c'està-dire la Campanie autrefois peuplée de Samnites. Les contraintes de la métrique alliées à une certaine affectation poussent souvent cet auteur à utiliser des termes parfois inattendus.

5 La traduction ne peut rendre le style recherché et les prouesses de versification de Gilles de Corbeil, qui utilise notamment de nombreux synonymes pour développer chaque thème. Il 
mène décrit par un médecin attentif aux «cas cliniques» rencontrés lors de son séjour salernitain, mais aussi arrière-plan moral et religieux teinté de superstition.

\section{Les sources de Gilles de Corbeil}

\section{Sources médicales directes}

Gilles de Corbeil n'est pas le premier médecin à évoquer ce phénomène. Un de ses maîtres à penser, Matheus Platearius ${ }^{6}$, auquel il se réfère fréquemment dans le traité des médicaments, paraît bien être sa source directe. Nous trouvons en effet dans le Liber iste attribué à cet auteur le passage suivant concernant la thériaque:

Bue avec du jus d'armoise elle provoque les menstrues; bue avec du jus de poireau, elle expulse le foetus mort et l'animal engendré dans la matrice des femmes de Salerne. Le fait monstrueux est que lorsqu'il sort vivant, s'il touche la terre, immédiatement l'enfant meurt. C'est pourquoi les Salernitaines tenant des bâtons pour le tuer l'empêchent de toucher le sol. ${ }^{7}$

D'autres auteurs salernitains, vraisemblablement antérieurs à Matheus Platearius, ont également écrit sur ce phénomène: d'abord Copho, auteur d'une anatomie du porc mais aussi d'une Practica qui indique au chapitre De matrice:

Nombreuses dans cette région sont celles qui, après l'accouchement, souffrent et sont en danger à cause d'une rétention de sang menstruel ou à cause d'un animal appelé arpa. ${ }^{8}$

Autre auteur, Iohannes Platearius, qui dans sa Practica apporte les précisions suivantes au chapitre De retentione menstruorum:

Note que les remèdes qui conviennent pour provoquer les menstrues font sortir le placenta et le foetus mort et le crapaud (bufonem) frère des salernitains. Note que les femmes de Salerne, au début de la conception et surtout quand le foetus doit être vivifié s'efforcent de tuer ce frère en buvant du jus d'ail et de poireau. ${ }^{9}$

Il s'agit là de traités de la pratique médicale destinés essentiellement à donner des conseils thérapeutiques aux médecins. Cependant, un autre type

est à remarquer que de Corbeil n'emploie jamais le terme de arpa que nous trouverons chez de nombreux auteurs pour désigner le fruit monstrueux. En revanche, ce terme figure dans les rubriques marginales du manuscrit unique contenant le De uirtutibus ... (Londres B. L. Add. 22 399), manuscrit copié au début du XIII ${ }^{\mathrm{e}}$ siècle.

6 Matheus Platearius est vraisemblablement l'auteur du Circa instans, ouvrage traitant des simples qui eut un succès considérable durant tout le Moyen Age, et d'un commentaire à l'Antidotarius magnus de Salerne connu sous le nom de Liber iste. Cf. Ausécache 2007.

7 D'après le manuscrit Winchester College, Warden and Fellows Library 24, f. 19va.

8 De Renzi IV, 501sq.

9 Paris, BnF lat. 15115, f. 260. Cependant, dans le manuscrit BnF lat. 6954 qui contient également la Practica de Platearius, la mention du crapaud est remplacée par celle de l'arpa. 
d'ouvrage fait également allusion à ce phénomène: certains commentaires à l'Isagoge $e^{10}$, datables de la première moitié du XII ${ }^{\mathrm{e}}$ siècle, mentionnent cette naissance en s'efforçant de fournir des explications d'ordre théorique. Ces commentaires se trouvent au chapitre Distancia inter masculum et feminam, qui insiste surtout sur la différence existant entre l'homme et la femme en matière de complexion, de tempérament: chaud et sec pour l'homme, froid et humide pour la femme. La naissance monstrueuse est alors considérée comme le résultat de l'excessive humidité féminine. Un manuscrit d'Oxford ${ }^{11}$ comporte ces précisions:

les femmes longobardes, lorsqu'elles sont enceintes, ont une abondance de sang menstruel supérieure à ce qui est utile au foetus. Ce qui reste dans la matrice génère un animal qui est appelé crapaud (crapallus) ${ }^{12}$, qui sort en même temps que l'enfant libérant ainsi la mère. Cependant, s'il reste il ronge et provoque la mort; certains pensent qu'on peut le tuer par une consommation fréquente d'herbes, ce qui fait que ces femmes sont plus pâles que les autres.

Ce commentaire anonyme est le premier texte faisant référence à un groupe ethnique, les Longobards. Cette mention se retrouve également dans les commentaires de Bartholomaeus et Musandinus, sans doute légèrement postérieurs ${ }^{13}$. Cependant, les deux auteurs donnent à l'«animal» le nom de arpa et une variante transforme occidere en accidere ce qui peut alors se traduire par: «certains pensent que cela se produit à cause d'une consommation fréquente d'herbes», cette pratique expliquant alors la génération du monstre et non sa destruction ...

Ces différentes assertions de médecins que nous qualifierons volontiers de «salernitains» se situent dans un arrière-plan intellectuel qu'il nous faut préciser afin de mieux comprendre leur mode de pensée.

\section{Les conceptions médicales}

La théorie de la conception qui sous-tend ce type de discours est l'héritage des théories d'Hippocrate et Galien: l'embryon est le résultat de l'union des

10 Isagoge Iohannitii, version latine abrégée par Constantin l'Africain des Questions sur la médecine de Hunain ibn Ishaq. Cf. Jacquart/Micheau 1996, 101sq. Les commentaires salernitains, notamment à l'Isagoge, marquent le passage à une médecine plus théorique qualifiée de physica. L'Isagoge est un des éléments d'un corpus de textes (souvent accompagné de commentaires) connu depuis la Renaissance sous le nom d'Articella. Cf. Kristeller 1976; Jordan 1987.

11 Oxford, Bodleian Lib. Digby 108, f. 4-26. Ce manuscrit contient l'un des plus anciens commentaires après ceux de Paris, BnF lat. 544, et Chartres (manuscrit aujourd'hui disparu). Merci à Irene Caiazzo pour ces informations et la primeur de son article à paraître.

12 Le terme crapallus utilisé ici semble indiquer une origine française de l'auteur.

13 Erfurt Ampl. 4 175, f.14a pour Bartholomaeus (cf. Lawn 1979, 286). Winchester 24, f. 35b pour Musandinus. 
deux semences, masculine et féminine, union qui se fait dans la matrice ${ }^{14}$. Dès que cette union a eu lieu, la matrice se referme hermétiquement pour permettre le développement de l'enfant qui est alors nourri par le sang ordinairement expulsé par les menstrues. Cependant, dans un certain nombre de cas (matrice mal refermée, surplus de sang menstruel), une seconde conception semble possible par «superfétation», phénomène qui explique notamment les naissances multiples ${ }^{15}$. L'avenir du deuxième embryon ainsi conçu diffère selon les cas. Ainsi, dans le traité De la superfétation attribué à Hippocrate, peut-on lire:

Quand la superfétation a lieu chez une femme, si le premier enfant est dans le milieu de la matrice, le produit de la superfétation tombe expulsé par l'ancien fœtus. Mais si le premier enfant est dans l'une des cornes, la femme met au monde en dernier lieu le produit non viable, après que la matrice s'est relâchée et humectée, étant délivrée du produit viable. Si le produit de la superfétation ne sort pas aussitôt, il cause de la douleur, un flux fétide et de la fièvre; le visage, les jambes, les pieds enflent, ainsi que le ventre; anorexie jusqu'à ce qu'il soit expulsé. [...] Les produits de la superfétation, si, n'ayant pas encore les membres distincts ils ne sont qu'une chair, ne se gonflent pas, mais se putréfient jusqu'à ce qu'ils sortent. ${ }^{16}$

Cette description peut correspondre au phénomène décrit par les médecins de Salerne, avec une difficulté cependant: Gilles de Corbeil précise bien que le produit «dégénéré» sort avant l'enfant et non après. On peut néanmoins penser que les conceptions d'Hippocrate ont largement influencé les Salernitains, qui désignaient volontiers leur ville comme la Civitas hippocratica.

Cependant, au-delà des conceptions anatomiques et physiologiques, intervient également le point de vue des médecins sur la sexualité féminine. Celui-ci n'est pas dénué des considérations misogynes d'ordre moral et religieux qui imprègnent l'ensemble de la société médiévale ${ }^{17}$. En effet les médecins médiévaux, majoritairement clercs, ont adopté la vision héritée de l'Antiquité selon laquelle la sexualité féminine est débridée et insatiable ${ }^{18}$. Une œuvre anatomique du XII ${ }^{\mathrm{e}}$ siècle, l'Anatomia Ricardi, précise même les choses:

Le désir et le plaisir du coït sont situés à l'entrée de la matrice [...] la partie supérieure cependant est infatigable et insatiable quant à son appétit. ${ }^{19}$

14 La position d'Aristote qui nie le rôle de la semence féminine a surtout de l'influence à partir du XIII ${ }^{\mathrm{e}}$ siècle. Sur les théories de la conception ayant cours au XII siècle, voir notamment Jacquart/Thomasset 1985, 73-108; Van der Lugt 2004, 43-59.

15 Dasen 2005, 33.

16 Littré 1853, 477.

$17 \mathrm{Au} \mathrm{XII}{ }^{\mathrm{e}}$ siècle, citons entre autres Hildebert de Lavardin qui présente la femme comme un véritable fléau ou André le Chapelain faisant au livre III du De amore un catalogue des défauts féminins. Cf. Puig Rodriguez-Escalona 1995, 158; Baldwin 1994, 17.

18 On doit à Juvénal, évoquant Messaline, la célèbre formule: «[...] fatiguée de l'homme mais rassasiée non pas» (Sat. VI, 130). Gilles de Corbeil prend plaisir à développer ce thème en comparant l'appétit sexuel de la femme au Tartare, au feu grégeois inextinguible (II, 234sq.). Selon lui, c'est la raison pour laquelle la femme serait poussée fréquemment à l'adultère si elle n'était pas retenue par la crainte d'une naissance (III, 158-164).

19 Sudhoff 1927, 231sq. 
Les médecins s'efforcent de comprendre ce phénomène physiologique: certains comparent la femme au bois humide long à s'enflammer mais brûlant plus longtemps. D'autres pensent que la matrice froide et humide recherche la chaleur du sperme. Dans le Pantegni $i^{20}$ qui a une grande influence sur les auteurs salernitains, le plaisir féminin est décrit comme étant plus fort que celui de l'homme puisque provoqué par l'émission des deux semences masculine et féminine ${ }^{21}$. Cependant, ces assertions d'ordre médical sont marquées par d'autres types de considérations.

\section{Morale chrétienne et préjugés}

Plusieurs points du récit de Gilles de Corbeil font référence à des principes d'ordre moral. L'idée qu'une naissance monstrueuse puisse être le fait d'un châtiment divin remonte à l'Antiquité et est reprise par les auteurs chrétiens pour lesquels toute créature est inscrite dans le dessein de Dieu. La faute dénoncée par un tel événement est d'emblée pensée comme étant de l'ordre d'un dérèglement sexuel ${ }^{22}$. Or, en terme de dérèglement sexuel, les femmes italiennes semblent avoir particulièrement mauvaise réputation: pratiques «sodomites» des femmes de marchands italiens à l'aide d'instruments appropriés, fausse virginité des jeunes épouses napolitaines ${ }^{23}$ et, selon Gilles de Corbeil, caractère éminemment volage des «samnites» si facilement séduites par un étranger de passage ... Ces femmes pécheresses sont donc particulièrement susceptibles de donner naissance à des monstres.

De plus, le corps féminin est objet de curiosité, incompréhension, peurs. Ainsi en est-il des nombreuses croyances populaires relatives aux menstrues: caractère extrêmement nocif du sang menstruel capable d'empoisonner plantes et animaux, de détériorer les métaux, de transmettre la rage; danger que représente la femme menstruée capable de ternir les miroirs par son seul regard ou de transmettre la lèpre lors d'un accouplement. S'il s'agit avant tout de croyances populaires, les lettrés s'en font le relais, dans les ency-

20 Traduction de Constantin l'Africain de l'ouvrage du médecin persan connu en Occident sous le nom d'Haly Abbas (al-Magusi), cet ouvrage divisé en dix livres de théorie et dix livres de pratique inspirés de Galien est longtemps resté une référence essentielle pour les médecins. Il fut éclipsé par l'introduction du Canon d'Avicenne. Cf. Jacquart 1995, 180sq.

21 Jacquart/Thomasset 1985, 113.

22 Il est également admis que des maladies puissent être provoquées par une sexualité non conforme à la morale. C'est ainsi que la lèpre est souvent considérée comme «un signe divin», châtiment résultant de rapports sexuels illicites (avec une femme lépreuse ou en période de menstruation ...). Cf. Jacquart/Thomasset 1985, 252-255.

23 Jacquart/Thomasset 1985, 210sq. 
clopédies notamment ${ }^{24}$. Le discours médical quant à lui transmet l'idée du caractère «animal» de la matrice capable de se déplacer jusqu'à suffoquer la malade, la «suffocation de la matrice» étant largement abordée dans tous les traités de pratique médicale avec les traitements destinés à la remettre en place.

Pour les auteurs qui comparent le fruit monstrueux à un crapaud (bufo ou crapallus), ce qui n'est pas le cas, rappelons-le, de Gilles de Corbeil, la référence semble également entachée de croyances dont certaines fort anciennes. En effet, depuis l'Antiquité les grenouilles, «symbole de la matière primitive, humide, informe», sont au cœur de mythes souvent liés à la fécondité, à la vie sexuelle féminine. Depuis le Néolithique, les offrandes de «Dames grenouilles» en relation avec la procréation sont nombreuses dans une aire géographique étendue, l'embryon dans l'utérus étant parfois identifié avec une grenouille ${ }^{25}$. Cependant le batracien est ambivalent, il peut être négatif voire maléfique, notamment lorsqu'il est associé à des pratiques magiques, à des déesses dangereuses, aux fléaux de l'Apocalypse ${ }^{26}$.

Au Moyen Age, le crapaud est associé à la pestilence, à la pourriture puis à la sorcellerie. Il est assimilé aux vers, aux serpents, et autres animaux rampants. Sa laideur, son caractère humide et tumescent, sa relation à la terre en font l'animal «idéal» pour désigner le fruit monstrueux sorti des entrailles d'une femme soupçonnée du péché de $\operatorname{chair}^{27}$. Le crapaud a la réputation de se nourrir de la terre ${ }^{28}$, ce qui explique peut-être pourquoi l'animal ne doit pas toucher le sol nu dont il pourrait se nourrir, se trouvant ainsi vivifié à son contact $^{29}$. De plus, la matrice féminine caractérisée par sa très forte humidité est elle-même souvent comparée à un animal humide du type poulpe, grenouille ou crapaud, animaux parfois représentés sous forme d'ex-voto destinés à faciliter l'accouchement ${ }^{30}$.

24 Tel Vincent de Beauvais dans le Speculum naturale, cité dans Jacquart/Thomasset 1985, 102. Les médecins ont alors des conceptions physiologiques qui font de la femme «une machine dangereuse». Cf. Thomasset 1992,363.

25 Lévêque 1999, 48-66.

26 Lévêque 1999, 92-94.

27 Il fait également partie des animaux qui tourmentent les femmes luxurieuses damnées; dans certains récits, il sort même du sexe de la femme ... Cf. Berlioz 1999a, 224.

28 Cf. Berlioz 1999b, 270.

29 L'héritage antique semble également présent dans cette crainte: à Rome, le nouveau-né monstrueux n'était pas enterré, «car mettre en terre un enfant mort, c'était réensemencer symboliquement la grande génitrice pour qu'il renaisse en un frère plus heureux; or cette graine maléfique, il ne fallait pas lui donner occasion de germer à nouveau. Pour éviter que le corps touche la terre, on le portait vite au fleuve». Cf. Gélis 1984, 361.

30 Cf. Gélis 1984, 126; Dasen 2002, 176, 186. 


\section{Particularités salernitaines}

Gilles de Corbeil évoque les femmes «samnites», ses sources directes Copho et Platearius mentionnent les «femmes de Salerne», mais les commentaires à l'Isagoge introduisent le terme de «longobardes» qui mérite d'être explicité. Les Longobards, peuple germanique installé en Italie du sud au VII ${ }^{\mathrm{e}}$ siècle, dominent la région jusqu'à la conquête normande terminée en 1077. Ils sont connus alors par l'ouvrage de Paul Diacre (VIII ${ }^{\mathrm{e}} \mathrm{s}$.), Historiae langobardorum, qui raconte notamment un stratagème par lequel des femmes de la tribu des Winniles se seraient fait passer pour des hommes en attachant leurs longs cheveux autour de leur visage à la façon d'une «longue barbe» pour remporter la victoire contre les Vandales $(\mathrm{I}, 8)^{31}$, ce qui aurait valu à ce peuple le nom de Langobardi (des mots germaniques lang et bart). Plus loin (I, 15), Paul Diacre raconte l'histoire d'une prostituée qui accoucha de sept enfants qu'elle jeta dans une mare pour s'en débarrasser. L'unique survivant devint un valeureux guerrier qui combattit avec succès la championne des Amazones $^{32}$. Les femmes longobardes ont donc de quoi étonner et même effrayer: confusion des sexes ${ }^{33}$, vie dissolue à l'origine de naissances multiples, cruauté d'une femme qui ne recule pas devant l'infanticide ... un tel récit a pu marquer durablement les esprits.

Gilles de Corbeil, quant à lui, s'intéresse surtout à Salerne et à sa population. Plusieurs passages du De uirtutibus ... font l'éloge de Salerne, éloge qui va du site de la ville qualifié d'exceptionnel (III, 478-487) à la qualité de la lutte menée contre la maladie (III, 469-477). Il voue un très grand attachement à cette cité et évoque avec tristesse le sac de la ville par les armées de l'empereur Henri VI en 1194 (III, 508-511). Dans l'exposé concernant le «frère de Salerne», il se fait l'écho d'une opposition apparemment très grave entre les villes de Salerne et Bénévent, accusant même cette dernière d'être à l'origine du fléau.

On ne voit pas bien l'argument médical d'une telle assertion; en revanche, Gilles de Corbeil semble épouser la vieille rancœur qui oppose les Salernitains aux Bénéventains depuis les guerres civiles qui ont provoqué le partage de l'ancien duché de Bénévent au IX ${ }^{\mathrm{e}}$, Salerne devenant principauté et siège épiscopal en 849. Cependant, tout en étant le thuriféraire de Salerne, son regard est bien celui d'un observateur étranger: les femmes «samnites» sont facilement séduites par un francus, terme qui semble désigner ici une origine

31 Ed. 1993, 22-24. Trad. française 1994, 17.

32 Ed. 1993, 28-30. Trad. française 1994, 20.

33 Thomasset 1993, 113: «cette confusion des sexes, cette transgression, aura des conséquences durables: les productions monstrueuses sont nombreuses chez les femmes lombardes». 
et non le statut; de plus il est bien précisé que c'est le pater italicus qui se réjouit de la naissance du monstre. Cette réaction peut surprendre, puisque l'«animal» est perçu comme étant le résultat d'une relation adultère mais, en contre-partie en quelque sorte, le père semble alors assuré que l'enfant normal est bien un enfant légitime, ce qui paraît donc être l'essentiel ${ }^{34}$. On pense en effet qu'en cas de naissances multiples le fruit qui sort en premier est celui qui a été placé le dernier dans la matrice ${ }^{35}$. Ainsi, le «frère de Salerne» qui cumule le fait de sortir en premier et de présenter un caractère monstrueux est-il interprété comme l'évidence même de la faute: la femme profitant de sa grossesse a eu des relations adultères, mais le phénomène de superfétation a déjoué son stratagème en révélant sa culpabilité.

Ainsi nous voyons que les médecins salernitains du XII ${ }^{\mathrm{e}}$ siècle vivent dans un contexte intellectuel et moral complexe, fondé sur des théories médicales héritées de l'Antiquité et fortement marqué par le poids des préjugés et superstitions. Cependant, nous relevons également dans leurs écrits, au-delà de la relation de faits étranges, la volonté de trouver des explications et des remèdes au mal. Pour Gilles de Corbeil, la recherche des causes de la maladie est toujours fondamentale; c'est la capacité de mener à bien cette démarche qui différencie le vrai médecin formé à la théorie du simple empiricus. Au plan des explications médicales, Gilles de Corbeil mentionne l'altération du sperme, l'absorption d'herbes ${ }^{36}$, des menstrues trop abondantes ou un dérèglement de la matrice qui nécessiteront des traitements à base d'épices, herbes et aromates. Dans cette dernière partie de l'exposé, l'explication religieuse du phénomène et le nécessaire culte rendu à Saint Matthieu (saint patron de la ville) semblent cités pour mémoire.

Gilles de Corbeil écrit son traité à la fin du XII ${ }^{\mathrm{e}}$ siècle et la valeur de son récit est qu'il représente le plus long et le plus complet témoignage sur ce phénomène qui semble localisé dans le temps et dans l'espace et n'est rap-

34 La question de la légitimité de l'enfant est bien sûr importante dans tous les peuples, mais peut-être est-il fait allusion ici à la spécificité des lois lombardes qui s'appliquent encore à cette époque pour certaines populations d'Italie du sud où «le principe de la personnalité des lois fut toujours respecté». Cf. Taviani-Carozzi 1996. La préservation de la lignée et la transmission du patrimoine familial paraissent alors l'emporter sur toute autre considération. On peut peut-être y voir également un souvenir du monde romain où certaines femmes semblaient profiter de leur grossesse pour avoir des relations adultères, certaines qu'elles étaient de ne pas compromettre la lignée. Macrobe, auteur tardo-antique qui eut une grande influence au Moyen Age, fait dire à une femme dans les Saturnales (II, 5, 9): «Je ne prends de passager que lorsque la cale est pleine.» Il s'agit alors d'une forme bien spécifique de la «chasteté» considérée seulement du point de vue de la sauvegarde de la lignée. Cf. Quignard ${ }^{2} 2004,28$.

35 Cf. Gélis 1984, 374.

36 Ce qui peut s'entendre de deux façons: soit une alimentation déficiente essentiellement végétale, soit des procédés abortifs à base de plantes. 
porté que par un petit groupe de médecins dans des traités de médecine pratique «généraliste»: en effet, nous ne trouvons aucune trace d'un tel cas dans les textes plus anciens ou contemporains précisément consacrés aux «maladies des femmes» tels la Gynaecia de Muscio ${ }^{37}$ ou l'ensemble de textes connu sous le nom de Trotula ${ }^{38}$.

\section{La fortune du «frère de Salerne» au Moyen Age}

De nombreux auteurs vont s'intéresser aux siècles suivants à ce phénomène en le commentant, parfois avec un certain embarras, et le plus souvent avec le désir de comprendre et d'expliquer. Nous pouvons saisir des variantes dans le raisonnement en fonction du contexte intellectuel de l'auteur et surtout des sources utilisées.

\section{La survie des théories salernitaines}

Les théories salernitaines, triomphantes au XII ${ }^{\mathrm{e}}$ siècle, l'«Age d'or» de la «Cité hippocratique», sont certes concurrencées aux siècles suivants, mais elles demeurent cependant présentes dans bon nombre d'ouvrages. Les auteurs qui suivent la tradition salernitaine représentée par Platearius et Gilles de Corbeil associent le plus souvent la génération de l'«animal» à celle d'un enfant normal.

Au tournant des XII ${ }^{\mathrm{e}}$ et $\mathrm{XIII}^{\mathrm{e}}$ siècles se constituent, notamment en Angleterre, des collections de Questiones à contenu médical et scientifique largement influencé par les doctrines salernitaines, elles-mêmes infléchies par la réception d'œuvres d'Aristote devenues depuis peu accessibles en latin (Physica, De generatione et corruptione). Une de ces questiones ${ }^{39}$ porte sur l'origine de l'arpo: «Pourquoi l'arpo est-il généré par le sperme?» Une fois la question posée, vient la définition:

37 Il s'agit d'un traité de pratique à l'usage des accoucheuses, ouvrage composé au VI ${ }^{\mathrm{e}}$ s. à partir de textes de Soranos (éd. Rose 1882). Cf. Maire 2004.

38 Ensemble de trois textes consacrés aux maladies et soins des femmes (éd. Green 2001). Dans le De curis mulierum attribuable à Trota, on trouve le passage suivant (par. 170): «Certaines femmes ont des morceaux de chair (frustra carnis) qui sortent de la matrice. Cela leur arrive à cause du sperme retenu à l'intérieur et devenu visqueux parce qu'elles ne se lavent pas après le coït. Nous leur faisons toujours des fomentations d'herbes chaudes.» Green 2001, 136. Mais rien n'indique que ces morceaux de chair soient vivants ni surtout qu'ils soient générés en même temps qu'un enfant. Merci à M. Green pour ses précieux conseils.

39 Question N12, Lawn 1979, 285-287. 
C'est un animal rampant (vermis) comparable à un crapaud; il est généré dans la matrice et naît avec le fœtus. Parce que cela arrive surtout dans certaines régions comme la Lombardie et l'Apulée, l'arpo est appelé leur frère par dérision.

Le phénomène est rapproché d'autres prodiges comme l'œuf de coq ou la vivification des poils d'animaux. Ensuite vient la réponse à la question:

Le sperme est hétérogène, il y a en lui une substance pure et une substance impure [...] ce qui est impur va à l'arpo, ce qui est pur va au fœetus.

Girardus Bituricensis ${ }^{40}$, dans son commentaire au Viaticum ${ }^{41}$, reprend largement les thèmes salernitains du XII ${ }^{\mathrm{e}}$ siècle, avec cependant une variante: la présence de l'animal (pecus) qui chez les Lombards est appelé arpia peut provoquer la rétention du placenta. Le phénomène se produit chez ceux qui comme les Lombards ${ }^{42}$ se nourrissent de fruits et d'herbes. L'animal est comparable à un crapaud et est appelé frère des Lombards car ils naissent toujours jumeaux ${ }^{43}$.

Rufinus (fin XIII ${ }^{\mathrm{e}}$ s.) décrit les effets de la Thériaque en se basant sur les ouvrages salernitains, mais en apportant deux variantes: c'est le fœtus mort qui est l'animal que l'on trouve dans la matrice des femmes de Salerne ou d'Apulie. Plus loin, sans paraître gêné par la contradiction de son propos, il indique que le crapaud (grapaldus) doit être tué sans toucher le sol sous peine de voir l'enfant né ensuite mourir de la lèpre. Rufinus reconnaît qu'entendre le récit d'un tel phénomène peut provoquer la stupeur mais, dit-il, cela est attesté par de nombreux sapientes salernitani $i^{44}$.

40 Médecin parisien d'origine berrichonne (début XIII ${ }^{\mathrm{e}} \mathrm{s}$.) connu pour son commentaire du Viaticum dont sont conservés de nombreux manuscrits. Cf. Jacquart-Micheau 1990, 156.

41 Le «Viatique du voyageur» de Ibn al-Gazzar, médecin kairouanais du $\mathrm{X}^{\mathrm{e}}$ siècle, fut introduit en Andalousie par un de ses élèves. Il s'agit d'un manuel pratique, les maladies étant classées en 7 chapitres de la tête aux extrémités. Cet ouvrage traduit par Constantin occupe une place prépondérante dans l'enseignement de la médecine, lu et cité par les maîtres salernitains, commenté à partir du XIII ${ }^{\mathrm{e}}$ siècle. Le chapitre 17 du livre VI concerne uniquement l'expulsion de la «secondine».

42 Le glissement de Longobards à Lombards peut signifier simplement l'abandon d'un terme ancien mais c'est bien ce terme de Lombards qui désigne alors ceux qui font commerce de l'argent et ont à ce titre une fort mauvaise réputation. Les femmes lombardes sont sujettes aux conséquences de leurs dérèglements sexuels, alors que les hommes sont souvent qualifiés de «lâches, rusés, traîtres» dans la littérature. Cf. Thomasset 1992, 365. Plus tard, on fera d'eux des spécialistes de l'empoisonnement.

43 D'après le manuscrit Paris BnF n.a.l.1701, f. 102vb.

44 Ed. Thorndike 1946, 316sq. 


\title{
Une autre théorie: la môle
}

D'autres auteurs rapprochent cette anomalie d'une maladie connue sous le nom de môle ou fausse grossesse, maladie décrite dès l'Antiquité, notamment dans le traité hippocratique Des maladies des femmes:

\begin{abstract}
Voici la cause de la formation d'une môle: quand les menstrues étant abondantes reçoivent une semence peu copieuse et morbide, il n'y a pas conception régulière, le ventre paraît plein comme chez une femme enceinte; mais rien ne remue dans le ventre; il ne se forme point de lait dans les mamelles, qui sont cependant turgescentes. Cet état dure deux ans, quelquefois même trois $[\ldots]$. D'abord on fera une fumigation générale; puis on prescrira un lavement qui produira un flux abondant de sang; car peut-être on mettra en mouvement la concrétion qui paraît être un embryon, par l'effet du médicament qui aura échauffé le ventre. ${ }^{45}$
\end{abstract}

Ce terme de môle qui, remarquons-le, ne figure pas dans les textes salernitains ${ }^{46}$ est abondamment employé à partir du XIII ${ }^{\mathrm{e}}$ siècle dans les commentaires au Traité des animaux d'Aristote. En effet, les commentateurs s'intéressent à la môle et à ses différentes causes: conception normale interrompue à cause de la mauvaise qualité du sang menstruel ou du sperme, surabondance de sang menstruel qui permet à la femme de développer une môle sans intervention de sperme masculin. Il peut également s'agir d'une émission de semence féminine lors d'un rêve érotique. La môle ainsi conçue par la seule semence féminine représente alors «la limite des capacités reproductives autonomes de la femme». Le sperme masculin étant absent, le résultat ne peut être qu'imparfait, inachevé, informe puisque c'est le sperme masculin qui est prédominant dans la formation de l'embryon et notamment de l'âme sensitive ${ }^{47}$. Certains de ces auteurs font le rapprochement entre le phénomène décrit par les Salernitains au XII ${ }^{\mathrm{e}}$ siècle et la môle et vont jusqu'à les identifier sans les mettre cependant en relation avec la venue d'un enfant.

Albert le Grand dans ses Questiones super De animalibus reprend l'idée d'une pollution nocturne et définit la môle comme une masse de chair générée par la seule semence féminine sans sperme masculin et privée de ce fait de forma sensitiva. Cette masse se nourrit du sang menstruel comme le fœtus. Ensuite, marquant une certaine réserve semble-t-il (ut dicitur et audivi), il rapporte que dans certaines régions, en même temps que la môle est toujours produit un crapaud (bufo), car cette matière est très apte à la génération d'un crapaud ${ }^{48}$. Ainsi le produit de cette génération inachevée est-il en

45 Ed. trad. E. Littré, VIII, 1853,149-151.

46 Il ne se trouve pas dans le Trotula mais figure dans la Gynaecia de Muscio, où la maladie est brièvement décrite: «la maladie appelée môle est une obstruction de la matrice à partir d'une fermentation précédente; elle s'étend souvent à l'ensemble du ventre de sorte que cela ressemble à une grossesse». Ed. Rose 1882, 65.

47 Cf. Van der Lugt 2004, 124-127.

48 Ed. Filthaut 1955, Livre X, Q.5, 216-218. 
quelque sorte «dédoublé», le crapaud étant alors un sous-produit de la môle. L'ensemble peut être considéré comme le fruit d'une sexualité féminine incontrôlée, la femme payant par la génération de ce monstre le prix de sa lascivité.

La môle est présente également dans Placides et Timeo (fin XIII ${ }^{\mathrm{e}} \mathrm{s}$.), ouvrage présentant sous forme d'un dialogue didactique, un état des connaissances de l'époque. Timeo explique la génération de la môle en reprenant l'idée de la pollution qui fait que les femmes rendent sperme non mie naturel mais mauvais et que dans la matrice se forme alors ne mie bonne char, car le matere est mauvaise, si ne peut faire bonne ouvre produisant une molla qui est ronde, rude, orde, doubteuse a veoir. L'expulsion d'un tel produit dégénéré est plus douloureuse qu'un accouchement, puisque nature n'œuvre pas les voies par termes, ainsi comme fait au droit fruit ${ }^{49}$.

Les théories se contredisent parfois, mais elles donnent en règle général une image négative de la femme aussi bien sur le plan moral que sur le plan physiologique, même si certains médecins prennent en compte les conditions de vie des femmes sujettes à ce genre de troubles. C'est notamment l'approche de Bernard de Gordon au chapitre De mola matricis (Livre VII, chap.18) de son Lilium medicine (1305):

La môle est une masse de chair énorme et sans forme; certains l'appellent pecus, d'autres lui donnent le nom de frater lombardorum, d'autres encore la nomment arpia. Elle est générée lorsque une grande quantité de semence féminine chaude parvient à la matrice alors qu'il y a une carence de semence virile; cela ne permet pas la formation d'un embryon mais produit un globus enormis qui fait croire à une grossesse. Cette maladie est très difficile à soigner et peut durer quatre années et même jusqu'à la fin de la vie sans guérison possible. Parfois sort une énorme masse de chair, beaucoup de sang ou bien un grand nombre de ventosités. Il faut comprendre que des vers, des reptiles, arpia et frater lombardorum peuvent être générés dans la matrice mais les vers se trouvent plutôt dans les intestins ${ }^{50}$. En revanche, quelque chose d'énorme qui peut être appelé mola ou arpia peut être généré du fait de la corruption de la semence féminine, de la carence de semence masculine et de la chaleur non naturelle. On dit que cela se produit plus souvent chez les femmes de Lombardie parce qu'elles travaillent beaucoup (un grand nombre d'entre elles étant dépendantes) ou bien à cause d'une mauvaise alimentation comme en Apulie parce qu'il est dit communément que les Italiennes vivent mal, se nourrissant de fruits et de plantes, mais qu'elles sont bien vêtues ... 51

49 Ed. Thomasset 1980,147-150.

50 Un autre médecin de Montpellier, Jean de Tournemire, trace quant à lui un portrait plus précis: «la môle prend parfois la forme d'un animal monstrueux que les laïcs appellent arpa parce qu'il a des griffes (arpas) et un museau pointu comme une souris». Cf. Van der Lugt 2004, 126sq.

51 Ed. Lyon 1574, 643. Ce médecin dénonce l'alimentation quasi végétarienne des Italiennes comme une des causes de leur mauvaise santé; cependant il faut voir qu'il peut s'agir également d'une forme de topos littéraire, les habitants de la région de Naples étant longtemps désignés par l'appellation de «mangeurs de feuilles», mangiafolia. Cf. Sereni 1981, 296-300. Merci à Bruno Laurioux de m'avoir fait connaître cet article. 
Le «frère des Lombards» se trouve encore mentionné au $\mathrm{XV}^{\mathrm{e}}$ siècle par Valescus de Tarenta ${ }^{52}$ dans sa Practica seu Philonium (c. 1418) au livre VI, chapitre De mola matricis. Pour faciliter l'expulsion de la môle, après avoir utilisé potions, bains, onguents, pessaires et fumigations,

... que la femme éternue fortement et se comprime comme si elle accouchait d'un fœtus, que sorte le frère des Lombards et qu'il aille à la potence (ad furcas) [...]. Il faut comprendre que ce sont les choses liquides et humides qui rendent la matrice flexible et souple et permettent que cet animal fait de chair, arpia ou frère des Lombards, soit expulsé [...] de même que des pessaires irritants permettant de se débarrasser de la môle ou arpia qui est un monstre contraire aux lois de la nature. ${ }^{53}$

Ainsi, pour tous ces médecins, «frère des Lombards», arpia, môle sont-ils des termes désignant le même phénomène qui ne semble pas impliquer l'existence d'un fotus en même temps, position qui était celle des Salernitains. Cependant, au XIV ${ }^{\mathrm{e}}$ siècle, le médecin Michele Savonarola ${ }^{54}$ établit une différence entre les deux phénomènes et ajoute d'autres explications. Au chapitre De mola matricis de sa Practica maior, il reprend l'idée que la môle est le produit de la seule semence féminine (pollution nocturne, sperme masculin non retenu lors d'un coït ou de mauvaise qualité). Le produit de cette conception est alors informe et dur comme de l'argile pétrifié par le feu. Ensuite, il présente un autre cas:

Et remarque que ce qui paraît impossible pour nos femmes est cependant véridique et cela s'est parfois produit dans notre cité (je le tiens de ma mère, femme digne de foi, qui en fut témoin): il arrive parfois aux femmes des régions méridionales et australes ainsi qu'à celles qui, du fait d'un régime de vie médiocre, ont un sang abondant et de mauvaise qualité, de mettre au monde en plus d'un véritable fœetus un morceau de chair vivant ayant la forme d'un animal que les femmes appellent bête sauvage (feram); la cause de cela n'est pas la qualité du sperme masculin émis en premier puisqu'à partir de lui le fœtus est déjà conçu, mais un égarement du second sperme émis lors d'un second coït, égarement provoqué par l'influence de la constellation ou de la vertu céleste comme le dit Aristote dans le livre 18 des animaux. Ainsi dans certaines régions d'Apulie (mon père en fut témoin), des femmes accouchent en même temps que d'un fœtus d'une bête, hibou, épervier, aigle, lézard; ces femmes affirment que lorsque sort avec le fœtus une bête ou un animal noble, alors le fœtus fut conçu avec un homme noble. Si la bête est rustique comme le hibou ou le lézard le foetus a été conçu avec un homme rustique et vulgaire et cette bête parfois tue le fœtus. Et on dit qu'elle a un tel pouvoir que si, tombant à terre au moment de l'accouchement, elle mord le fœetus, celui-ci meurt immédiatement. Cela est vrai malgré les apparences parce que nous n'avons pas l'habitude de voir de telles choses et, de même que dans les intestins peuvent naître des vers, ainsi dans la matrice peuvent être générés des animaux car il s'y trouve une matière apte à cela. ${ }^{55}$

52 Médecin montpelliérain, sans doute d'origine portugaise, auteur d'un traité sur la peste et d'une Practica populaire jusqu'à la fin du XVII' siècle. Cf. Fischer 1920, 175; Sarton 1948, III (2), 1199; Dulieu 1975, I, 209sq.

53 Ed. Lyon 1490, 213-213v.

54 Né vers 1384 d'une vieille famille de Padoue, il fit ses études de médecine à Padoue puis Ferrare. Il est l'auteur d'une Practica maior en latin et d'un traité Ad mulieres Ferrarienses en italien. Cf. Fischer 1920, 175; Sarton 1948, III (2), 1197.

55 Ed. Venise 1547, 276vb-277a. 
On le voit, Michele Savoranola reprend les thèmes salernitains: superfétation donnant lieu à une double conception, pouvoir maléfique de la bête. Cependant, il ne reprend pas le vocabulaire ancien pour désigner l'animal et apparemment le phénomène se produit également à Padoue. Autre particularité, l'intervention de la conjonction astrale qu'il semble être le premier à faire intervenir. Cependant, nous remarquons chez cet auteur comme chez Rufinus ou Albert le Grand un certain malaise à évoquer un tel événement. Rufinus évoquait les sapientes salernitani pour appuyer ses dires, Michele Savonarola se réfère au témoignage de ses parents.

Au début du XVII ${ }^{\mathrm{e}}$ siècle, le médecin montpelliérain Joannes Varandeus parle à plusieurs reprises de la môle dans son ouvrage De morbis et affectibus mulierum ${ }^{56}$. Au chapitre De mola et monstris, il précise que certaines môles sont associées à un véritable fœetus, ce qui constitue une difficulté supplémentaire pour la malade et le médecin ${ }^{57}$. Plus loin, au chapitre consacré à l'accouchement, il explique que lorsqu'une môle est associée étroitement à un fœtus, elle doit absolument sortir en même temps que lui, car en faisant obstacle elle peut provoquer la mort de l'enfant. Il rappelle alors qu'une môle associée à un fotus était appelée par certains médecins du passé frater Longobardorum parce qu'il est dit qu'un tel type d'accouchement est fréquent dans la région de $\mathrm{Milan}^{58}$. On le voit, les théories du XII ${ }^{\mathrm{e}}$ siècle ont la vie dure.

\section{Essai d'interprétation}

Que penser de ces descriptions qui, même lorsqu'elles diffèrent dans certains détails, semblent tourner autour du même phénomène? On peut partager le sentiment de L. Choulant, éditeur des poèmes médicaux de Gilles de Corbeil, écrivant en note à propos du «frère de Salerne» qu'il s'agit d'une chose satis obscura ... La prudence s'impose effectivement en matière d'explication mais du moins pouvons-nous essayer quelques pistes.

56 Cf. Diepgen 1925,73.L'œuvre de ce médecin, constituée de ses cours consignés par ses élèves, a été publiée après sa mort survenue en 1617. Cf. Dulieu 1979, II, qui écrit à son propos: «L'ensemble manque d'originalité mais Varanda n'avait pas cherché autre chose qu'à enseigner à ses élèves l'état des connaissances médicales de son temps» (353).

57 Ed. Lyon 1620, livre II, chap. 5, 88-99.

58 Ed. Lyon 1620, livre II, chap. 7, 134sq. 


\section{Un avortement?}

On pense bien sûr d'abord aux cas d'avortement provoqué. Depuis Hippocrate, la pratique de l'avortement est attestée dans de nombreux textes médicaux, de façon plus ou moins explicite. Nombre de médicaments destinés à provoquer les menstrues sont également considérés comme des remèdes abortifs. Cela est visible dans les textes cités à propos de la thériaque qui, bue avec du jus d'armoise, provoque les menstrues, alors que bue avec du jus de poireau, elle provoque l'expulsion du fœtus mort ou de l'«animal». Dans la Practica de Iohannes Platearius, il est dit clairement que les femmes s'efforcent de tuer ce «frère» en buvant du jus d'ail et de poireau. Le commentaire à l'Isagoge contenu dans le manuscrit d'Oxford indique également que le crapallus peut être tué par une consommation fréquente d'«herbes». On peut donc penser que certains cas décrits correspondent à des pratiques abortives et que «l'imaginaire de la monstruosité et l'explication scientifique ne serviraient qu'à masquer une réalité plus inavouable» ${ }^{59}$.

Sans doute pouvait-il s'agir également d'un avortement spontané fréquent notamment chez les femmes des campagnes soumises à de durs travaux surtout pendant les mois d'été. Les carences alimentaires aggravent encore la situation et «les années de disette sont aussi des années de fausses couches» ${ }^{60}$. C'est peut-être ainsi que nous pouvons interpréter le témoignage de Bernard de Gordon parlant des femmes dépendantes et mal nourries. Cependant, le texte de Gilles de Corbeil pose une autre question: il dit clairement que le «frère de Salerne» accompagne l'enfant normal, partageant avec lui «l'abri de la matrice» et qu'il sort en premier au moment de l'accouchement. D'autres auteurs, nous l'avons vu, reprennent ce point de vue en soulignant le caractère extrêmement dangereux du phénomène.

\section{Une réelle anomalie de la grossesse?}

Que peut-il y avoir de médical dans la description faite par les auteurs salernitains et leurs successeurs? N'y a-t-il pas dans ces étranges récits une part de cas cliniques réels observés avec étonnement et incompréhension? L'accouchement reste longtemps une affaire exclusivement féminine, les textes salernitains précisent bien que ce sont les «femmes de Salerne» qui en sont les ordonnatrices. Il se peut que des anomalies de la grossesse, des difficultés lors de certains accouchements, mal comprises et mal interprétées, soient à

59 Jacquart/Thomasset 1985, 224.

60 Gélis 1984, 305. 
l'origine du récit devenu par la suite quasiment mythique ${ }^{61}$. Le médecin n'intervient pas et ses réflexions et conseils thérapeutiques sont élaborés à partir des textes de ses prédécesseurs et de cas qui lui sont rapportés. La médecine savante relaie les récits des femmes, avec une certaine gêne parfois, tout en s'efforçant de trouver une explication au phénomène.

\section{Une légende?}

Cependant, la part de l'irrationnel et des préjugés, importante dans les différentes relations médiévales de l'«événement», perdure bien au-delà du Moyen Age et dans des milieux sans doute plus éduqués que celui des «femmes de Salerne»: au XIX ${ }^{\mathrm{e}}$ siècle, dans le Dictionnaire des Sciences médicales se trouve la liste des médecins ayant «publié de semblables absurdités». Ces «absurdités» sont les dires des sages-femmes qui prétendaient avoir vu

... des femmes accoucher tantôt d'animaux morts tels que des rats, des taupes, des tortues, tantôt d'animaux vivants à quatre pieds, armés de griffes ou d'ongles crochus (harpies). Elles assurent que les moles [...] jouissent en naissant de la faculté de marcher dans la chambre; que d'autres peuvent voler, qu'elles cherchent quelquefois à se cacher, même à rentrer dans la matrice de laquelle elles sont sorties ... ${ }^{62}$

Visiblement certaines sages-femmes et certains médecins du XIX ${ }^{\mathrm{e}}$ siècle se faisaient encore l'écho des vieilles croyances médiévales. L'article précise également que les anciens médecins utilisaient des méthodes violentes pour expulser la môle, et dénonce au passage un médecin montpelliérain qui semble alors encore user des mêmes méthodes. L'auteur de l'article pourfend donc les anciennes doctrines mais pense tout de même que «la fréquence de l'approche conjugale peut également faire périr l'embryon; aussi les moles s'observent assez fréquemment chez les jeunes époux nouvellement mariés».

Ainsi, dans ce domaine les légendes semblent-elles vivaces, y compris dans les milieux informés. Tout ce qui touche à la conception et à la naissance est resté longtemps entaché de mystère et de crainte, tant on se trouve alors à «la frontière poreuse entre animalité et humanité» ${ }^{63}$. Le «frère de Salerne» semble bien se situer dans cette zone mouvante où l'imagination se nourrit vraisemblablement de l'incompréhension de certains phénomènes. En

61 L'abondante littérature médicale consacrée à des anomalies telles que la mort in utero d'un jumeau, le placenta praevia, la môle hydatiforme (phénomène rare mais qui suscite de nombreux articles) est souvent accompagnée d'illustrations impressionnantes pour le néophyte et il est loisible d'imaginer l'étonnement mêlé d'effroi que de tels cas ont pu susciter dans l'entourage des parturientes. Cf. par exemple Speert 1973; Pons/Charlemaine/Papiernik 2000; Baergen 2005.

62 Panckoucke 1819, t. 34, 5.

63 Barras 2004, 102. 
matière d'accouchement, l'empirisme a longtemps prévalu, faisant parfois intervenir une dimension irrationnelle, un recours au divin, aux pratiques magiques ${ }^{64}$. Dans ce domaine comme dans d'autres touchant par exemple à la pharmacopée, se rencontrent médecine savante et médecine populaire. Le moment de la naissance conjugue appréhension, douleur, risque. Le moindre événement venant en perturber le déroulement normal peut être interprété et amplifié, l'imaginaire entourant la naissance l'emportant parfois sur la rationalité.

\section{Sources imprimées}

Anatomia Ricardi, éd. K. Sudhoff, Archiv für Geschichte der Medizin 19 (1927) 209-239

Collectio Salernitana, éd. S. de Renzi (Naples 1852-1857)

de Corbeil, Gilles, Carmina medica, éd. L. Choulant (Leipzig 1826)

- De uirtutibus et laudibus compositorum medicaminum, éd. M. Ausécache, thèse de doctorat E. P. H. E. (Paris 2003, en cours de publication)

de Gordon, Bernard, Lilium medicine (Lyon 1574)

de Tarenta, Valescus, Practica seu Philonium (éd. Lyon 1490)

Diacre, Paul, Historiae langobardorum, éd. L. Capo, Paolo Diacono, Storia dei Longobardi (Milan 1993); trad. fr. F. Bougard, Histoire des Lombards (Turnhout 1994)

le Grand, Albert, Questiones super De animalibus, éd. E. Filthaut, Opera omnia, t. 12 (Münster 1955)

Hippocrate, Opera omnia, éd. trad. fr. E. Littré, 10 vol. (Paris 1839-1861)

Isagoge Iohannitii, éd. G. Maurach, «Isagoge ad Techne Galieni», Sudhoffs Archiv B. 62/2 (1978) $148-174$

Muscio, Gynaecia, éd. V. Rose, Sorani gynaeciorum vetus translatio latina (Leipzig 1882)

Placides et Timeo ou li Secrés as philosophes, éd. C. Thomasset (Genève 1980)

Questiones salernitane, éd. B. Lawn, The Prose Salernitan Questions. An Anonymous Collection Dealing with Science and Medicine Written by an Englishman c.1200, with an Appendix of Ten Related Collections (Oxford 1979)

Rufinus, Herbarius (éd. L. Thorndike, The Herbal of Rufinus, Chicago 1946)

Savonarola, Michele, Practica (Venise 1547)

Trotula, éd. M. Green, The Trotula, a Medieval Compendium of Women's Medicine (Philadelphie 2001)

Varandeus, Joannes, De morbis et affectibus mulierum (Lyon 1620)

\section{Bibliographie}

Ausécache, M., «Un Liber iste, des Liber iste? Un Platearius, des Platearius? Etat des lieux d'un projet d'édition», La Scuola Medica Salernitana. Gli autori e $i$ Testi, Actes du colloque de Salerne, 3-5 Novembre 2004 (Florence 2007) 1-30

Baergen, R. N., Manual of Benirschke and Kaufmann's Pathology of the Human Placenta (New York 2005)

Baldwin, J. W., The Language of Sex: Five Voices from Northern France around 1200 (Chicago 1994)

Barras, V., «La naissance et ses recettes en médecine antique», dans: Dasen, V. (éd.), Naissance et petite enfance dans l'Antiquité, Actes du colloque de Fribourg, 28 novembre- $1^{\text {er }}$ décembre 2001 (Fribourg-Göttingen 2004) 93-102

Berlioz, J., «Le saint, la femme et le crapaud», dans: Revel, J./J. C. Schmitt (dir.), L'ogre historien. Autour de Jacques Le Goff (Paris 1999a) 223-242

64 Coulon 2004, 221-225. 
- «Le crapaud, animal diabolique: une exemplaire construction médiévale», dans: Berlioz, J./ M. A. Polo de Beaulieu (dir.), L'animal exemplaire au Moyen Age, $V^{e}-X V^{e}$ s. (Rennes 1999b) 267-288

Caiazzo, I., «Un inedito commento sulla Isagoge Iohannitii conservato a Parigi», La Scuola Medica Salernitana. Gli autori e i Testi, Actes du colloque de Salerne, 3-5 Novembre 2004 (Florence 2007) 93-123

Coulon, G., «Images et imaginaire de la naissance dans l'Occident romain», dans: Dasen, V. (éd.), Naissance et petite enfance dans l'Antiquité, Actes du colloque de Fribourg, 28 novembre$1^{\text {er }}$ décembre 2001 (Fribourg/Göttingen 2004) 209-225

Dasen, V., «Métamorphoses de l'utérus d'Hippocrate à Ambroise Paré», Gesnerus 59 (2002) 167-186

- Jumeaux, jumelles dans l'Antiquité grecque et romaine (Zürich 2005)

Diepgen, P., «Der <frater Salernensis und Lumbardorum»», Archiv für Geschichte der Medizin XVI (1925) 73sq.

Dulieu, L., La médecine à Montpellier, 6 tomes (Avignon 1975-1997)

Fischer, J., «Der «frater Salernensis und Lumbardorum», Archiv für Geschichte der Medizin XII (1920) 173-175; XVI (1925) 73

Gélis, J., L'arbre et le fruit: la naissance dans l'Occident moderne (XVI-XIX $\left.{ }^{e}\right)$ (Paris 1984)

Jacquart, D., «La scolastique médicale», dans: Grmek, M. D. (dir.), Histoire de la pensée médicale en Occident. 1. Antiquité et Moyen Age (Paris 1995) 175-210

- /C. Thomasset, Sexualité et savoir médical au Moyen Age (Paris 1985)

- /F. Micheau, La médecine arabe et l'Occident médiéval (Paris 1996)

Jordan, M. D., «Medicine as science in the early commentaries on Johannitius», Traditio 43 (1987) 121-145

Kristeller, P. O., «The school of Salerno: its development and its contribution to the history of learning», Studies in Renaissance Thought and Letters, vol. I (Rome 1956) 495-551

- «Bartholomaeus, Musandinus and Maurus of Salerno and other early commentators of the Articella with a tentative list of texts and manuscripts», Italia medioevale e umanistica 19 (1976) 57-87, Studies in Renaissance thought and letters, vol. III (Rome 1993) 403-429

- Studi sulla Scuola salernitana (Naples 1986)

Lévêque, P., Les grenouilles dans l'Antiquité. Cultes et mythes des grenouilles en Grèce et ailleurs (Paris 1999)

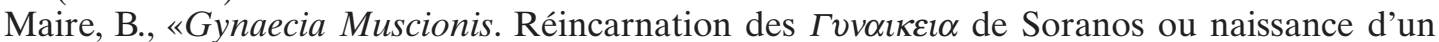
traité?», dans: Dasen, V. (éd.), Naissance et petite enfance dans l'Antiquité, Actes du colloque de Fribourg, 28 novembre-1 ${ }^{\text {er }}$ décembre 2001 (Fribourg/Göttingen 2004) 317-323

Panckoucke, C., Dictionnaire des sciences médicales, t. 34 (1819)

Pons, J. C./C. Charlemaine/E. Papiernik, Les grossesses multiples (Paris 2000)

Puig Rodriguez-Escalona, M., Poesia misogina en la edad media latina (ss. XI-XIII) (Barcelone 1995)

Quignard, P., Le sexe et l'effroi (Paris 22004)

Sarton, G., Introduction to the History of Science (Baltimore 1948)

Sereni, E., «Note di storia dell'alimentazione nel Mezzogiorno: i Napoletani da mangiafoglia a mangiamaccheroni», Terra nuova e buoi rossi, e alti saggi per una storia dell'agricoltura europea (Turin 1981) 292-371

Speert, H., Histoire illustrée de la gynécologie et de l'obstétrique (Paris 1973)

Taviani-Carozzi, H., La terreur du monde, Robert Guiscard et la conquête normande en Italie: mythe et histoire (Paris 1996)

Thomasset, C., «Aspects de la femme médiévale dans le Lilium medicinae de Bernard de Gordon», dans: Femmes-Mariages-Lignages, $X I I^{e}-X I V^{e}$ s. , Mélanges offerts à G. Duby (Bruxelles 1992) 361-372

- «Le corps féminin ou le regard empêché», Micrologus I (1993) 99-114

Van der Lugt, M., Le ver, le démon et la vierge. Les théories médiévales de la génération extraordinaire (Paris 2004)

Vieillard, C., Essai sur la société médicale et religieuse au XII siècle. Gilles de Corbeil, médecin de Philippe Auguste et Chanoine de Notre-Dame, 1140-1224, avec fac-similé de la Hierapigra (Paris 1908) 